\title{
Complexity and continuity of treatments among privately insured youth diagnosed with bipolar disorder
}

\author{
Sara Evans-Lacko ${ }^{1 *}$, Anne W. Riley ${ }^{2}$ and Susan dosReis ${ }^{3}$ \\ 1 Health Services and Population Research Department, Institute of Psychiatry, King's College London, London, UK \\ 2 Department of Population, Family and Reproductive Health, Johns Hopkins Bloomberg School of Public Health, Baltimore, MD, USA \\ ${ }^{3}$ University of Maryland School of Pharmacy, Baltimore, MD, USA \\ Department and Institution to which the work is attributed: Department of Health Policy and Management, Johns Hopkins Bloomberg School of Public Health
}

\section{Edited by:}

Stephan Collishaw, Cardiff University

School of Medicine, UK

Reviewed by:

Daniel Smith, Cardiff University, UK

*Correspondence:

Sara Evans-Lacko, Health Service and Population Research Department,

Institute of Psychiatry, King's College London, De Crespigny Park, London SE5 BAF, UK.

e-mail: sara.evans-lacko@iop.kcl.ac.uk
Objectives: To examine longitudinal patterns of complexity, continuity, and initiation of treatment for youth diagnosed with bipolar disorder. Additionally, we explore bipolar diagnosis stability and its relationship to observed treatment patterns. Methods: A cohort of 426 privately insured youth (ages 6-18) diagnosed with bipolar disorder was identified from the 2000-2001 Thomson/Medstat-MarketScan ${ }^{\circledR}$ database. Medication complexity was defined as number of different psychotropic medication classes dispensed during a 6-month period following a new treatment episode of bipolar disorder. Treatment continuity was examined over a 6-month follow-up period, specifically focusing on mood stabilizing medications and antidepressant monotherapy. Predictors of complexity and continuity were investigated. Results: Fifty-five percent of youth received more than one and $25 \%$ received three or more different types of psychotropic medication classes during follow-up. This was contrasted with several youth having no prescription fills $(21 \%)$ and $31 \%$ discontinuing mood stabilizing medication. Youth with a stable bipolar diagnosis were more likely to have continuity of mood stabilizing prescriptions (OR: 4.05), but also greater psychotropic medication complexity. Age, health status/comorbidity, and being in a managed care plan were also related to complexity and continuity of psychotropic medication class regimens. Conclusions: More evidence is needed on the causal patterns leading to increased psychotropic medication complexity and continuity and how diagnosis of bipolar disorder may drive treatment patterns.

\section{Keywords: bipolar disorder, children, adolescent, child psychiatry, psychotropic drugs, mood stabilizers, antidepressants,} medication adherence

\section{INTRODUCTION}

The pharmacological management of children and adolescents with bipolar disorder is widely recognized as challenging and complex. Consequently, youth receive multiple trials of medication, often resulting in the concomitant use of several different agents. In a survey of parents of youth aged 6-17 years old who had bipolar disorder, youth were taking on average $3( \pm 1.5)$ medications and had completed $6( \pm 3.7)$ previous trials of psychotropic medication (Bhangoo et al., 2003). Other population based studies of youth with bipolar disorder ranging from 0 to 20 years of age have confirmed high levels of polypharmacy (Jerrell and Shugart, 2004; Moreno et al., 2007; Jerrell, 2008).

Several factors have been implicated as contributing to high overall rates of medication use and the frequent medication trials among youth with bipolar disorder. For one, youth with bipolar disorder often have more psychiatric comorbidities than youth with other psychiatric disorders (Geller et al., 1994; Jerrell and Shugart, 2004; Birmaher et al., 2006; Moreno et al., 2007; Jerrell, 2008). The Journal of the American Academy of Child and Adolescent Psychiatry (JAACAP) established guidelines that advise treating each disorder separately because comorbidity worsens the prognosis for youth with bipolar disorder (Kowatch and DelBello, 2005). However, this is complicated because common treatments for comorbid disorders may include antidepressants and stimulants which the guidelines suggest may "exacerbate mania." Therefore, it is recommended to use antidepressants in combination with a mood stabilizing medication. These issues may be even more complicated for younger children who tend to have a less clear presentation of bipolar disorder (Ryan et al., 1987; Biederman et al., 2005; Wozniak, 2005; Goodwin and Jamison, 2007).

In addition to psychiatric comorbidity, lack of evidence regarding effective treatment options for youth with bipolar disorder is likely to influence high rates of psychotropic medication use found in previous studies (Carlson, 2005; Post and Kowatch, 2006; Danielyan et al., 2007). Consequently, clinicians will extrapolate from clinical trials, open trial, and case reports that involve adult patients (Leibenluft, 2006). It is possible that insufficient evidence and uncertainty around efficacy and tolerability of many psychotropic medications used to treat youth with bipolar disorder may lead to delays in initiation of treatment, frequent switching patterns, or discontinuity. Additionally, uncertainty may lead to inappropriate treatment provision. For instance, one study has shown low levels of guideline concordance among youth diagnosed with bipolar disorder, including about one-third of youth receiving antidepressant monotherapy (Evans-Lacko et al., 2010). Moreover, a recent study by Geller et al. (2010), showed that over an 8-year follow-up period, less than two-thirds of youth ever received a recommended antimanic medication. To date, however, there has been 
very little attention given to the sequence or utilization patterns of psychotropic treatment for youth with bipolar disorder as the majority of the literature has been based on cross-sectional studies, and so the field lacks knowledge of how these medications are being used over time. To understand these types of medication patterns, it is essential to examine longitudinal patterns of use. This study addresses this gap by examining longitudinal patterns of the complexity, continuity and initiation of psychotropic medication and psychotherapy treatment for youth diagnosed with bipolar disorder. Additionally, we explore the stability of services associated with bipolar disorder and its relationship to the observed treatment patterns. Finally, we investigate which factors predict continuity of pharmacological treatment. This project was approved by the Johns Hopkins University Bloomberg School of Public Health Institutional Review Board.

\section{MATERIALS AND METHODS DATA SOURCE}

Data come from the Thomson-Medstat MarketScan (2000-2001) database, a national dataset containing standardized, detailed, enrollee-specific clinical utilization information across inpatient and outpatient services, and prescription drug information from approximately 45 employer sponsored health plans. Information on filled prescriptions are recorded at the individual level allowing for investigation of drug use patterns relating to timing of diagnoses (Adamson, 2005). Outpatient pharmacy claim files consist of data from all settings including mail order and specialty pharmacies and manual and electronically submitted claims. Data from the outpatient pharmacy claim files that were used include enrollee-specific de-identified identification number, prescription dispensing date, quantity dispensed, and the National Drug Code (NDC). The NDC is a unique number assigned by the Food and Drug Administration that identifies the manufacturer, the specific medication name and strength, and the product package size. Since the medication name is not recorded on the pharmacy claims file, the Thomson-Medstat MarketScan database was linked to the Multum lexicon database using the NDC to extract the drug name (Mumford, 2008).

\section{SAMPLE CHARACTERISTICS}

Behavioral health claims of an initial cohort $(N=426)$ of privately insured youth 6-18 years of age who received at least two diagnoses (i.e., at least two visits associated with a diagnosis of bipolar disorder) or an inpatient hospitalization for bipolar disorder were examined. This method of case identification has been associated with a high positive predictive value (94\%) and modest sensitivity (80\%) (Lurie et al., 1992). The validity of this approach was also explored for bipolar disorder in adults and depression (Kashner, 1998; Unutzer et al., 2000; Blow et al., 2004).

The cohort with a new treatment episode was identified by excluding all youth receiving any type of bipolar disorder diagnosis during the first 4 months of the 24-month study period. Youth who were enrolled in the study period for less than six additional months following their initial diagnosis of bipolar disorder were also excluded due to insufficient follow-up information. Youth with co-occurring seizure disorders who may receive mood stabilizing medications for reasons other than treatment of bipolar disorder were excluded.
Enrollees with 2 years of continuous enrollment did differ from those with 1 year of continuous enrollment or less in terms of region of residence and plan type, with a higher proportion of those with 2 years of continuous enrollment living in the northeast and fewer living in the west compared to those with 1 year of continuous enrollment or less. Also, significantly more persons with 2 years of continuous enrollment were in an HMO and fewer in a Point of Service Plan (POS) compared to those with 1 year of continuous enrollment or less. There were; however, no differences among these groups by age or gender. This is not a probabilistically representative sample of the nation, but the data include a large sample of youth from all regions of the country and all types of private insurance plans.

Age was analyzed by the following age groups: 6-11, 12-14, 15-16, and 17-18 years. It is worth noting the rationale for combining 6- to 11-year-olds in one group because it is a wider range than the other age groups. Combining all the elementary schoolaged youth (6-11 years) still resulted in a small sample. To address concerns that the 6- to 9-year-olds $(N=33)$ are developmentally quite different from 10- to 11-year-olds $(N=38)$ and their patterns of use might be quite different, several analyses were conducted and showed no consistent pattern of differences between these subgroups, as described below.

\section{PSYCHOTROPIC MEDICATION MEASURES Complexity of psychotropic treatment}

Medications were organized into eight major psychotropic classes: stimulants, antidepressants, antipsychotics, antiparkinsonian, anxiolytics, and sedative/hypnotics, mood stabilizing anticonvulsants, and other antimanic agents (i.e., lithium). The complexity of psychotropic treatment was defined as the total number of different classes of psychotropic medication prescriptions filled 6 months following the initial diagnosis of bipolar disorder.

\section{Immediate vs. delayed initiation of psychotropic medication}

Youth were defined as initiating psychotropic medication immediately if they filled a prescription for the medication within 1 month of their initial diagnosis of bipolar disorder. Youth who filled a prescription for a psychotropic medication more than 1 month following initial diagnosis were considered to have delayed initiation.

\section{Continuity of psychotropic medication treatment}

Three mutually exclusive patterns were used to describe psychotropic medication continuity. These groups include: (i) Complete discontinuation, defined by the cessation of pharmacotherapy with no resumption during the remaining time in the 6 month follow-up period; (ii) Non-persistence, defined as discontinuation of medication at some point, but resumption of treatment within the 6-month follow-up period; and (iii) Continuity, defined as filling prescriptions for the same medications throughout the entire 6-month period.

\section{STABILITY OF BIPOLAR DIAGNOSIS}

Three patterns of care were used to categorize individual's stability of bipolar diagnosis (i) Youth who received a service claim associated with a diagnosis of bipolar disorder at least 5 out of the 6 months following their initial diagnosis were considered to have a stable 
pattern of diagnosis; (ii) Youth who received a service claim associated with bipolar disorder in fewer than 5 months, but received care for bipolar disorder in the 6th month of the follow-up period were considered to have an intermittent pattern; and (iii) Youth who stopped receiving claims for bipolar disorder were described as having a discontinuous pattern of diagnosis.

\section{HEALTH STATUS AND COMORBIDITY}

A youth's health status and co-existing medical and psychiatric morbidities were assessed using the Johns Hopkins Adjusted Clinical Groups (ACG) Case Mix System, Version 8.0. The ACG system is well-established, valid, and commonly used to adjust for comorbidity (Weiner et al., 1991; Johns Hopkins, 2001). Adjustments are made for age and gender to individuals with similar morbidity and anticipated healthcare resource consumption. Individuals are stratified into resource utilization bands (RUB) with scores ranging from 0 (none) to 5 (high) anticipated use of future healthcare resources. These categories have been empirically validated in the US and internationally and it has been demonstrated that people with higher RUB's have more severe comorbidity and use more healthcare care resources (Weiner et al., 1991; Johns Hopkins, 2001; Reid et al., 2001; Starfield, 2006).

\section{DENSITY OF PSYCHIATRISTS}

The 2001 Area Resource File (ARF) was used to obtain the availability of child psychiatrists in each county (US Department of Health and Human Services, 2004). The total number of child psychiatrists in the youth's county of residence was divided by the number of 6- to 18-year-olds living in the county (also obtained through the ARF) to construct the variable density of child psychiatrists for youth. ARF data were linked to the claims data using the county of residence for each youth.

\section{STATISTICAL ANALYSIS}

Descriptive statistics were conducted first to assess the overall 2-year treated prevalence of the more common psychotropic medications as well as the complexity and continuity of psychotropic treatment 6 months following the initial diagnosis of bipolar disorder. Results are also described by age group, sex, geographical region, and ACG RUB score. Estimates and 95\% confidence intervals were computed to characterize the magnitude of the difference between groups.

Logistic regression models with robust standard errors were constructed to compare the odds of continuous vs. non-continuous use of a mood stabilizer. Although antipsychotics are also indicated as a first line treatment for bipolar disorder, they were not included in this analysis because very few youth were using an antipsychotic without using a mood stabilizer $(N=28)$. Models include stability of bipolar diagnosis in addition to patient age, sex, RUB, density of psychiatrists, number of outpatient mental health visits, timing of psychotropic medication initiation (immediate vs. delayed), being enrolled in a managed care plan (vs. a fee for service plan), and use of psychotherapy as these factors are likely to influence the probability of being prescribed a mood stabilizer. Model building examined for collinearity, interaction, and confounding. All data were summarized using SAS version $9.1^{\circledR}$ (SAS Institute Inc., Cary, NC, USA).

\section{RESULTS}

\section{DESCRIPTIVE CHARACTERISTICS}

Youth had a mean age of 14.4 years. About half of youth were male (52\%). Twelve percent were from a rural area and $88 \%$ were from an urban/suburban area. More than three-quarters of youth (78\%) were enrolled in a managed care plan. Antidepressants were the most common type of prescription filled (61\%). Although psychotropic medication use/prescribing is common for this cohort of youth, there was also a substantial proportion who did not have any pharmacy claims for a psychotropic medication (14\%). Older youth (ages 17-18) were less likely to fill a prescription for any type of psychotropic medication and younger youth (ages 6-11) were more likely to have a prescription filled for a stimulant.

\section{COMPLEXITY OF PSYCHOTROPIC MEDICATION USE}

Complexity of psychotropic medication use was examined overall and by age group. Approximately $21 \%$ of youth did not receive any psychotropic medication and as many as $25 \%$ were prescribed more than three different classes of psychotropic medications over the 6 month follow-up period. The distribution of psychotropic medication use for 17- to 18-year-olds resembles a left skewed distribution, with the highest proportion receiving either no medications or one agent from a single psychotropic class (59\%). Few 17- to 18 -year-olds received four or more different classes of psychotropic medications (9\%). Prevalence rates for the 6- to 11-year-old group resemble a normal distribution, with several youth receiving multiple classes of psychotropic medication (62\%) and the mode number of medication classes being 2 . A higher proportion of youth aged 6-9 years old had no psychotropic prescriptions compared to youth aged $10-11$ years of age ( $23 \%$ vs. $5 \%$ ). However, a similar proportion aged 6-9 years as aged 10-11 years had four or more psychotropic prescriptions (10\% vs. 13\%).

\section{STABILITY OF BIPOLAR DIAGNOSIS AND COMPLEXITY OF PSYCHOTROPIC MEDICATION TREATMENT}

Table 1 describes the stability of bipolar diagnosis by demographic, clinical, and insurance plan characteristics. Most youth were categorized as having a discontinuous pattern of diagnosis (41\%); 38\% of youth received an intermittent pattern of diagnosis and $21 \%$ received a continuous pattern of diagnosis for bipolar disorder. There was little variation between diagnostic groups in terms of demographic characteristics; however, youth with more complex medication regimens were more likely to have a continuous pattern of bipolar diagnosis. Health severity (as measured by RUB score) was also associated with stability of bipolar diagnosis, in that youth who have a higher RUB score are more likely to have a continuous pattern of bipolar diagnosis.

Figure 1 shows the number of different classes of psychotropic medications taken by youth each month for 6 months following their initial diagnosis of bipolar disorder as a function of the stability of bipolar diagnosis. Of note, stability of bipolar diagnosis was not significantly associated with age $(p=0.89)$. No significant differences in number of classes of medication were observed between groups at month 1 ; but, at month 6 , youth with a continuous pattern of bipolar diagnosis were using more different types of classes of psychotropic medication classes. Youth with a continuous pattern of diagnosis also tended to increase the number of different classes 
Table 1 | Stability of bipolar diagnosis by demographic and clinical characteristics $(N=426)$.

\begin{tabular}{|c|c|c|c|}
\hline & $\begin{array}{l}\text { Discontinuous pattern, } N=175(41.1 \%) \\
{[\%(95 \% \text { confidence interval)] }}\end{array}$ & $\begin{array}{l}\text { Intermittent pattern, } N=162(38.0 \%) \\
{[\%(95 \% \text { confidence interval })]}\end{array}$ & $\begin{array}{l}\text { Stable pattern, } N=89(20.9 \%) \\
{[\%(95 \% \text { confidence interval })]}\end{array}$ \\
\hline \multicolumn{4}{|l|}{ AGE } \\
\hline $6-11(N=65)$ & $40.0(28.3-52.9)$ & $40.0(28.3-52.9)$ & $20.0(11.5-32.1)$ \\
\hline $12-14(N=109)$ & $40.4(31.2-50.2)$ & $37.6(28.7-47.4)$ & $22.0(14.9-31.2)$ \\
\hline $15-16(N=129)$ & $41.9(33.3-50.9)$ & $36.4(27.6-46.2)$ & $21.7(15.1-30.0)$ \\
\hline $17-18(N=123)$ & $41.5(32.8-50.7)$ & $39.0(30.0-48.9)$ & $19.5(13.1-27.8)$ \\
\hline \multicolumn{4}{|l|}{ GENDER } \\
\hline $\mathrm{M}(N=222)$ & $42.3(35.8-45.1)$ & $40.5(34.1-47.3)$ & $17.2(12.6-22.9)$ \\
\hline$F(N=204)$ & $39.7(33.0-46.8)$ & $35.3(28.8-42.3)$ & $25.0(19.3-31.6)$ \\
\hline \multicolumn{4}{|l|}{ RUB } \\
\hline Low $(1-3)(N=211)$ & $44.1(37.3-51.1)$ & $37.4(31.0-44.4)$ & *18.5 (13.6-24.5) \\
\hline $\operatorname{Med}(4)(N=168)$ & $35.7(28.6-43.5)$ & $42.3(34.8-50.1)$ & $22.0(16.2-29.2)$ \\
\hline High (5) $(N=47)$ & $46.8(32.4-61.8)$ & $25.5(14.4-40.6)$ & $27.6(16.1-42.9)$ \\
\hline \multicolumn{4}{|l|}{ MANAGED CARE } \\
\hline Fee for service $(N=95)$ & $49.5(39.1-59.8)$ & $30.5(21.7-40.9)$ & $20.0(12.8-29.7)$ \\
\hline Managed care $(N=331)$ & $38.7(33.4-44.2)$ & $40.2(34.9-45.7)$ & $21.2(17.0-26.0)$ \\
\hline \multicolumn{4}{|c|}{ USE OF PSYCHOTHERAPY } \\
\hline Yes $(N=162)$ & $42.0(34.4-50.0)$ & $35.8(28.5-43.7)$ & $22.2(16.2-29.6)$ \\
\hline No $(N=264)$ & $40.5(34.6-46.7)$ & $39.4(33.5-45.6)$ & $20.1(15.5-25.5)$ \\
\hline
\end{tabular}

*Statistically significant at the $p=0.05$ level.

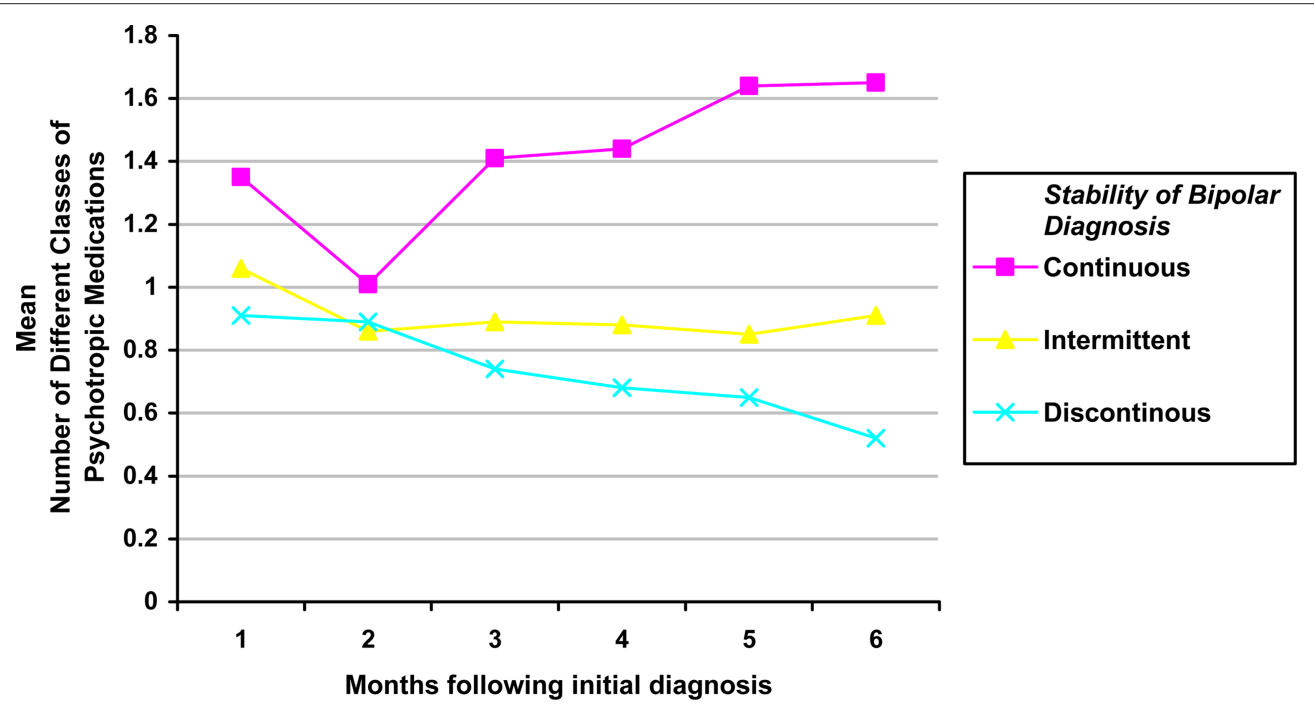

FIGURE 1 | Mean number of classes of psychotropic medication prescriptions filled by stability of bipolar diagnosis by month.

of prescriptions filled over the 6-month period, while youth in the intermittent or discontinuous group took the same number or fewer types of medications over the 6-month follow-up period.

\section{CONTINUITY AND TIMING OF INITIATION OF PSYCHOTROPIC MEDICATION TREATMENT}

Table 2 incorporates timing of initiation and continuity of psychotropic medication to describe five mutually exclusive patterns for utilization of mood stabilizing medication by demographic, clinical, and insurance plan characteristics. Statistically significant differences were found for age, use of psychotherapy, and stability of bipolar diagnosis. Those with a continuous pattern of diagnosis are significantly more likely to initiate and to have continuity of a mood stabilizer. Psychotherapy treatment was associated with delayed but more continuous use of a mood stabilizer. Regarding age, there seems to be a trend that older youth are less likely to ever fill a prescription for a mood stabilizer and also less likely to establish continuity of mood stabilizing medication. Younger youth, also seem to be less likely to have non-persistence of a mood stabilizing medication. 
Table 2 | Patterns of mood stabilizing prescription fills by demographic, clinical characteristics, and insurance plan characteristics ( $N=426$ ).

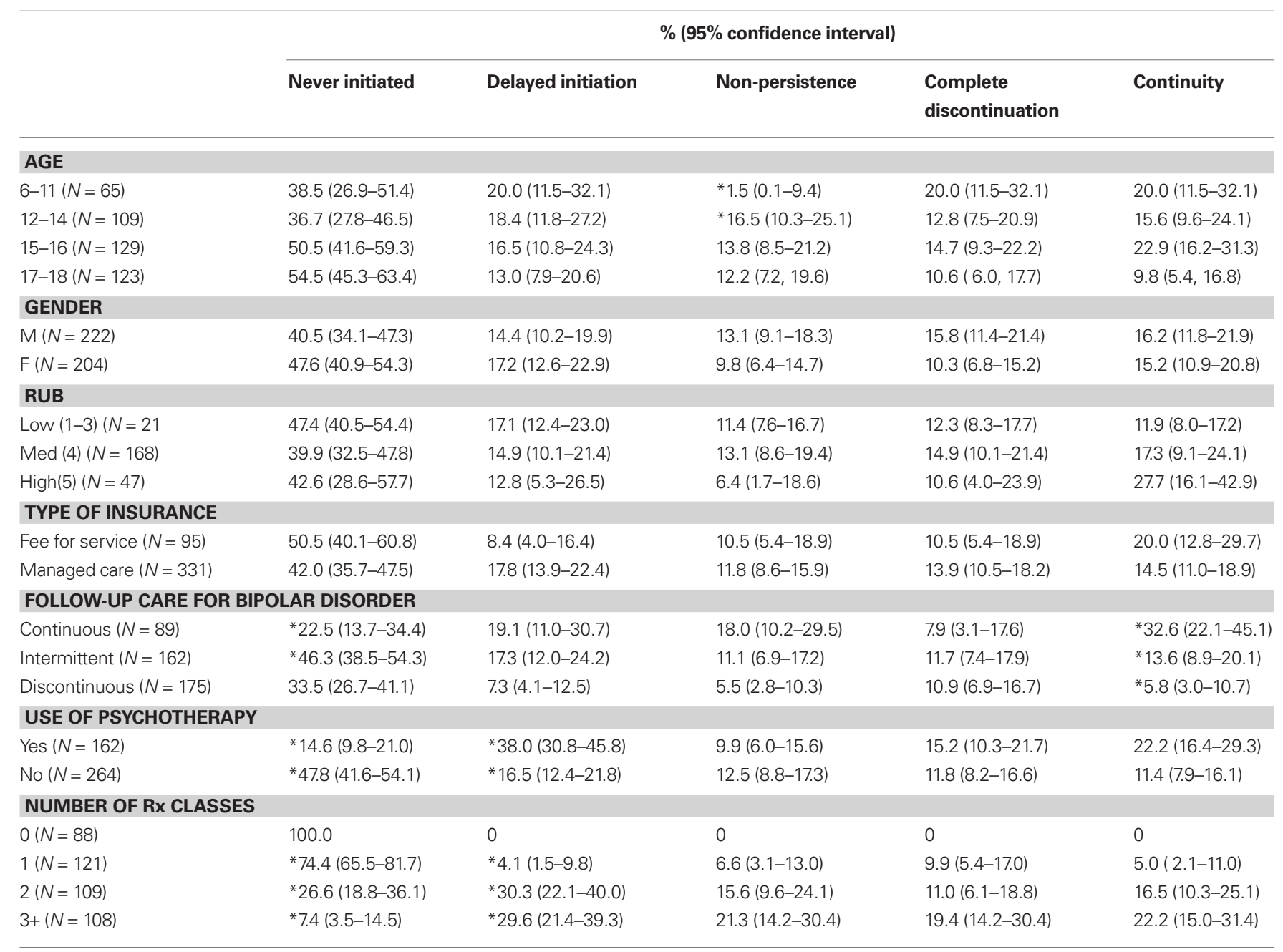

*Statistically significant at the $p=0.05$ level.

Figure 2 shows the continuity and persistence of a mood stabilizing medication for youth by stability of bipolar diagnosis. Youth with a continuous pattern of bipolar diagnosis are significantly more likely to receive a mood stabilizer in a continuous manner than youth with an intermittent or discontinuous pattern of diagnosis and the least likely to completely discontinue mood stabilizing medication. The group with a discontinuous pattern of bipolar diagnosis is also the most likely to completely discontinue mood stabilizing medication. Figure 3 shows the same relationship between continuity of bipolar diagnosis and continuity of pharmacological treatment, but for antidepressant monotherapy. Here a different pattern emerges with the group having a continuous stable pattern of bipolar diagnosis being the most likely to receive antidepressant monotherapy, which is not recommended. The group with a continuous stable pattern of bipolar diagnosis is still less likely than the discontinuous group to completely discontinue antidepressant monotherapy $(p=0.05)$, and is also less likely than the intermittent group to have non-persistent contraindicated antidepressant use $(p<0.05)$; but, there is no statistically significant difference in the continuity of antidepressant monotherapy between groups with differing patterns of diagnostic stability for bipolar disorder.

\section{MULTIVARIATE ANALYSES}

Table 3 shows the results of a logistic regression for the odds of receiving a mood stabilizer in a continuous manner by stability of bipolar diagnosis when controlling for demographic and service utilization characteristics. It appears that youth who are receiving a continuous pattern of bipolar diagnosis are more likely to receive continuous mood stabilizing prescriptions than youth with a discontinuous pattern of bipolar diagnosis. Delaying use of a mood stabilizer following initial diagnosis for bipolar disorder; however, did not predict later discontinuation/continuity of a mood stabilizer. This is true when controlling for age, sex, health severity/ comorbidity (as measured by RUB score), use of psychotherapy, density of psychiatrists, and insurance plan characteristics. Youth enrolled in a managed care plan and youth who were more ill (as measured by RUB score) were also significantly more likely to have continuity of a mood stabilizer or antipsychotic medication.

\section{DISCUSSION}

Complex medication regimens were common in youth diagnosed with bipolar disorder; approximately half of youth (55\%) received more than one and $25 \%$ of youth received three or more different 

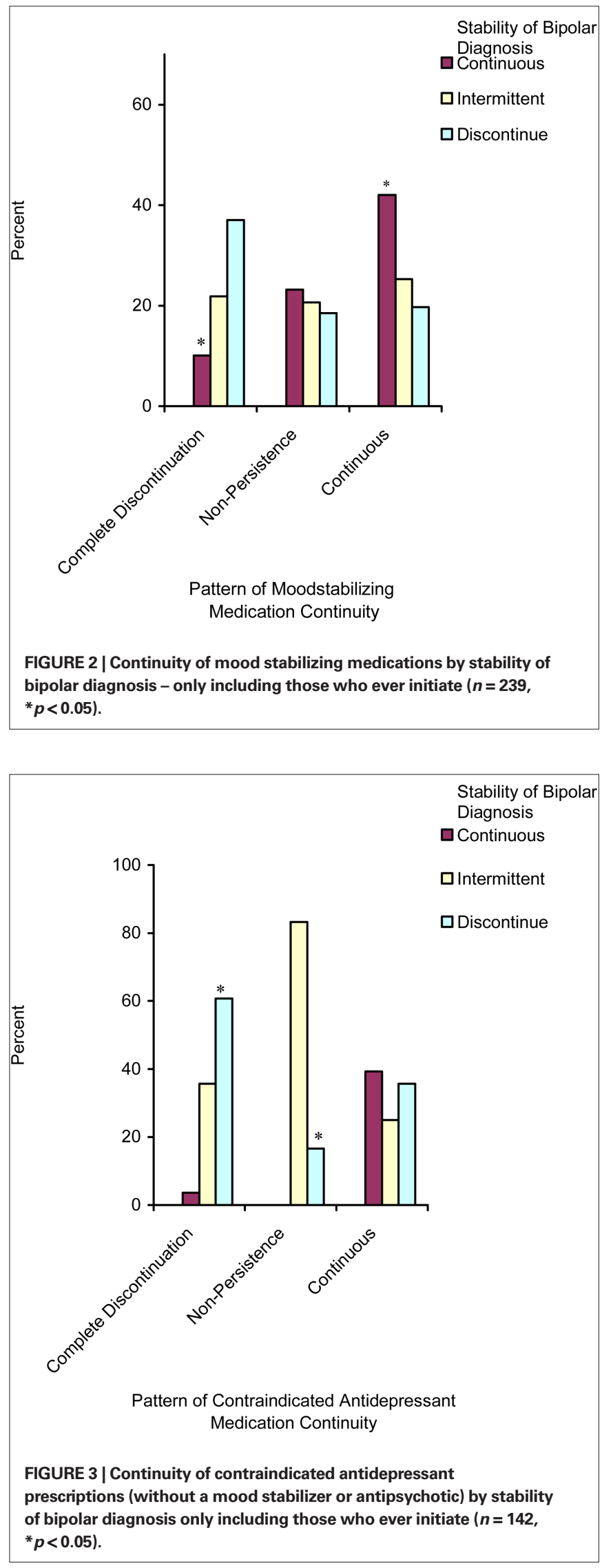

Table 3 | Logistic regression model for probability of continuity of mood stabilizing medication treatment by stability of bipolar disorder diagnosis over 6-month follow-up period ( $N=211$ ).

\begin{tabular}{lll}
\hline & Odds ratio & $\mathbf{9 5 \%} \mathbf{C l}$ \\
\hline \multicolumn{2}{l}{ STABILITY OF BIPOLAR DIAGNOSIS } & \\
$\begin{array}{l}\text { Discontinuous(ref) } \\
\text { Intermittent }\end{array}$ & $* 2.31$ & \\
Continuous & $* 4.05$ & $1.17-4.80$ \\
Number of mental outpatient & $* 1.04$ & $1.83-8.94$ \\
mental health visits & & $1.01-1.06$ \\
Immediate vs. delayed use & 1.12 & \\
Age & 0.95 & $0.59-2.13$ \\
SEX & & $0.85-1.06$ \\
F (ref) & & \\
M & 1.05 & $0.57-1.93$ \\
MANAGED CARE & & \\
Fee for service (ref) & & \\
Managed care & $* 2.25$ & $0.62-1.46$ \\
RUB (ref = low) & 0.95 & $0.55-1.37$ \\
Density of psychiatrists & 0.87 & \\
(per 1,000 youth) & & $0.46-11.50$ \\
Psychotherapy & 0.86 & \\
\hline
\end{tabular}

* Statistically significant at the $p=0.05$ level.

classes of psychotropic medications in the 6-month period following initial diagnosis of bipolar disorder. However, only $28 \%$ of youth continuously used a mood stabilizing medication in the 6-month follow-up period.

Despite high overall rates of psychotropic medication use, considerable variation in psychotropic medication continuity and complexity was apparent in this group of youth diagnosed with bipolar disorder. High overall rates of psychotropic medication use were contrasted with the finding that several youth had no prescriptions filled for psychotropic medication in the 6-month period following initial diagnosis of bipolar disorder. Of those who initiated a mood stabilizing medication, about $31 \%$ discontinued the medication within the 6-month follow-up period. Stability of bipolar diagnosis, age, health severity, and insurance plan characteristics were all shown to be important factors in describing differences in psychotropic medication complexity and continuity.

Older age had an interesting relationship with psychotropic medication complexity. The 17- to 18-year-old age group had the highest proportion with no psychotropic medications filled during the 6-month follow-up period and overall in the 2-year study period. This may be because this data only included information on filled prescriptions and it is possible that medications were prescribed but not filled. Therefore, this difference between age groups may reflect the fact that 17 - to 18 -year-olds are less likely to fill their prescriptions than youth of other ages. This is often a transitional age in which youth gain more independence in terms of their healthcare utilization. Although they still have insurance coverage, they might lack awareness regarding appropriate treatments and help-seeking or might be resistant to using certain psychotropic medications. Nevertheless, this might represent an 
important target group when thinking about further intervention. Other differences by age were not statistically significant except that younger youth (ages 6-11) were more likely to have a prescription filled for a stimulant. This is likely related to the fact that younger youth are also more likely to receive a diagnosis of ADHD (Geller et al., 2000).

Surprisingly, age was not statistically significantly associated with continuity of mood stabilizing medication in the multivariate analysis. This suggests that once youth were in care age did not affect medication utilization patterns. There were however, differences in age among ever initiating a mood stabilizer or antipsychotic and non-persistent patterns of use. First, youth who were less than 15 were significantly more likely to ever fill a prescription for a mood stabilizing medication in the 6-month follow-up period, than older youth ages $15-18(p=0.05)$. Moreover, older youth (12-18) were more likely to have a non-persistent pattern of mood stabilizing use compared to younger youth (6-11 years of age) ( $14 \%$ vs. $2 \%)$. Patterns of discontinuity for mood stabilizing medications were, however, similar among all age groups. Therefore, although we might expect older youth to have a more traditional phenotype of bipolar disorder and that, subsequently, they might be more easily treated and establish continuity of medication use, it is possible that lack of adherence is influencing these patterns.

This study also identified a relationship between stability of bipolar diagnosis and continuity and complexity of psychotropic medication treatment. Youth who received a continuous pattern of diagnosis for bipolar disorder were more likely to receive more complex and continuous psychotropic medication treatment regimens than youth for which the diagnosis was discontinued over the 6-month follow-up period. Although this may be related to the fact that youth with a continuous pattern of diagnosis are a sicker group, visit the provider more often, and thus may have more opportunities to be prescribed additional psychotropic medications in a continuous manner, this relationship remained significant after controlling for health severity and number of outpatient mental health visits. Youth who received a diagnosis of bipolar disorder in a continuous manner were also more likely to receive more complex medication regimens when controlling for number of outpatient mental healthcare visits. These findings suggest that physicians who strongly suspect a diagnosis of bipolar disorder in youth are more likely to continue to prescribe or add on additional medications over the 6-month follow-up period. One explanation for this finding might be that when the diagnosis of bipolar disorder is less clear, physicians might be more reluctant to prescribe additional psychotropic medications. Finally, the observation that delaying use of a mood stabilizing medication is not significantly associated with continuity of a mood stabilizing medication suggests that being in treatment for bipolar disorder in a continuous manner is a more important factor for maintaining continuity of treatment with a mood stabilizer than immediate initiation of a mood stabilizer. Of those who delayed initiation of a mood stabilizing medication, 37\% also delayed initiation of a second psychotropic medication. Twenty-seven percent of this group initiated psychotherapy without delay and only $7 \%$ did not take any other psychotropic medications. Therefore, although it seems a large proportion of youth are delaying initiation of a mood stabilizer, we can see that almost one-third of youth had already initiated psychotherapy, and may have experienced a delay to all types of psychotropic treatment.

Youth with a continuous pattern of bipolar diagnosis are more likely to establish continuity of a mood stabilizing medication emphasizing the connection between diagnosis and treatment and the potential for diagnosis to be driving treatment. This relationship, however, may also be due to the phenotypic presentation of the youth's disorder, to the confidence of the provider in making the diagnosis and prescribing appropriate treatment, or patient/ familial preferences regarding treatment. Although this study provides new information on the longitudinal patterns of medication continuity and complexity for youth with bipolar disorder, several unanswered questions remain surrounding the reasons youth are experiencing these different patterns of use. For instance, although stability of bipolar diagnosis is positively and significantly associated with continuity of mood stabilizing medication prescriptions, there are also a substantial proportion of youth who have a discontinuous or intermittent pattern of bipolar diagnosis, but are using mood stabilizers in a continuous manner. For some youth this may be due to a misclassification bias in that the youth may still have bipolar disorder, but they were not receiving care or a claim associated with bipolar disorder. Nevertheless, it is interesting to note that these youth continued to receive pharmacologic treatment for bipolar disorder but not a diagnosis for bipolar disorder. This finding is supported by previous research which shows a significant number of youth being prescribed antipsychotic, mood stabilizing and antidepressant medications without presence of an indicating diagnosis (Chen et al., 2009).

Moreover, we can not be exactly sure why youth discontinue services for bipolar disorder or mood stabilizing medication treatment. Patterns of medication use and continuity of claims for bipolar disorder may result from several factors. First, discontinuation of mood stabilizing medication or of treatment for bipolar disorder may be due to youth who were initially misdiagnosed with bipolar disorder. Discontinuation of mood stabilizing medication could either be the result of lack of tolerability or ceasing to fill prescriptions of that medication. Non-persistent use of medication may be due to non-adherence, but may also be due to switching providers. More complex medication regimens may be a result of treatments not resolving symptoms and physicians adding on additional medications, additional psychiatric comorbidities or physician practice style. Additionally some physicians may be afraid to remove a medication once it is initiated for fear of decompensation. Delayed initiation of a mood stabilizing medication following diagnosis of bipolar disorder may be the result of not seeing a provider immediately who is capable of prescribing a medication, reluctance of the provider to immediately initiate mood stabilizing medications, or the youth or parent deciding not to fill the initial prescription for a mood stabilizer. Finally, social, economic and cultural factors can also affect medication use and continuity.

There was also a significant positive relationship between contraindicated antidepressant use and stability of bipolar diagnosis. For youth with a stable pattern of bipolar diagnosis, the likelihood of ever initiating antidepressant monotherapy treatment in addition to maintaining continuous treatment with an antidepressant 
was not different for youth with a stable pattern of bipolar diagnosis vs. an intermittent or discontinuous pattern. Other research has also shown that a significant proportion of youth diagnosed with bipolar disorder receive antidepressant monotherapy (Moreno et al., 2007; Evans-Lacko et al., 2010). This suggests some level of uncertainty in terms of appropriate treatment regimen even for those with a continuous pattern of bipolar diagnosis. Youth with a continuous pattern of bipolar diagnosis were less likely to discontinue antidepressant monotherapy treatment compared to those with an intermittent or discontinuous pattern of bipolar diagnosis. This suggests a relationship between diagnostic uncertainty and patterns of medication use for all types of psychotropic treatment - even those that are contraindicated.

\section{LIMITATIONS}

There are some limitations to this research. Despite its strengths, claims data have limitations in that the validity of diagnoses cannot be verified and the data itself may include coding discrepancies. Factors such as misdiagnosis, inaccurate diagnosis or "up-coding" for reimbursement may influence diagnostic rates in utilization data. We do not mean to propose that all youth in this cohort are "true" bipolar cases, we aim to present the treatment and diagnostic patterns occurring in practice. Further research is needed to determine which youth discontinue diagnosis and/or treatment due to: misdiagnosis, barriers to care or result from an acute phase of the illness with subsequent recovery. Moreover, we do not have sufficient data to conclude why, in some cases, treatment for bipolar disorder continues when the diagnosis is discontinued. A substantial proportion of youth were included in the discontinuous diagnosis group and treatment patterns varied among individuals within this group. Although we characterize each subgroup to a degree, the data do not allow us to examine all of the potential predictors associated with each of the diagnostic and treatment patterns. We do not have data on some sociodemographic confounders such as education, income, race and ethnicity that in addition to undetected personal and familial factors may help to explain service utilization patterns. This study population excluded those without insurance and was not representative of those with insurance provided through some mechanism other than employer. These data, however, describe real world patterns of medication use by a cohort of children with a diagnosis of bipolar disorder.

\section{REFERENCES}

Adamson, D. M. (2005). Health Research Data for the Real World: The MarketScan Databases. Ann Arbor, MI: Thomson Medstat.

Bhangoo, R. K., Lowe, C. H., Myers, F. S., Treland, J., Curran, J., Towbin, K. E., and Leibenluft, E. (2003). Medication use in children and adolescents treated in the community for bipolar disorder. J. Child Adolesc. Psychopharmacol. 13, 515-522.

Biederman, J., Faraone, S. V., Wozniak, J., Mick, E., Kwon, A., Cayton, G. A., and Clark, S. V. (2005). Clinical correlates of bipolar disorder in a large, referred sample of children

Regarding the selection of the initial cohort, youth who did not receive a diagnosis for the first 6 months of the study period were considered to be newly diagnosed. However, it is possible that some youth received a diagnosis of bipolar disorder before this time period and erroneously were considered newly diagnosed. Nevertheless, these youth would be expected to receive continuous treatment upon reengagement of services. Additionally, because the data were based on pharmacy claims, we cannot be sure if it was the physician or parent/youth who discontinued the medication.

\section{CONCLUSIONS}

Many youth who receive two or more diagnoses of bipolar disorder and/or are hospitalized for that diagnosis do not continue to receive treatment for bipolar disorder over the 6-month period following diagnosis. Those who do receive a continuous diagnosis of bipolar disorder are more likely to receive continuous pharmacological treatment for bipolar disorder, but are also more likely to have complex regimes with multiple psychotropic agents/medications. Nevertheless, among those who continue to receive a diagnosis of bipolar disorder and associated care at least monthly, more than half do not consistently fill a prescription for a mood stabilizing drug and a similarly large proportion receive medications that are currently contraindicated according to AACAP and other guidelines. The difficulties of making this diagnosis accurately in youth and of treating early onset bipolar disorder most certainly contribute to the fact that most treatment for bipolar disorder in youth is not concordant with the treatment guidelines. At this point, we present only initial, descriptive trends and further exploration and investigation of predictive factors of continuity and discontinuity are needed. Nonetheless, this study indicates a high level of variability in treatment patterns among youth who are continuously insured may be useful in guiding policy and new research initiatives.

\section{ACKNOWLEDGMENTS}

I am thankful to my mentors including Drs. Donald Steinwachs, Elizabeth Kastelic, Kay Redfield Jamison, and Thomas Louis, for their helpful comments on design and interpretation of this study. This work was funded by an Institutional Training grant from the NIMH (T32 MH 19545). Thomson/Medstat provided this data to us free of charge as it was part of a dissertation.

Carlson, G. A. (2005). Early onset bipolar disorder: clinical and research considerations. J. Clin. Child. Adolesc. Psychol. 34, 333-343.

Chen, C. Y., Gerhard, T., and Winterstein, A. G. (2009). Determinants of initial pharmacological treatment for youths with attention-deficit/hyperactivity disorder. J. Child Adolesc. Psychopharmacol. 19, 187-195.

Danielyan, A., Pathak, S., Kowatch, R. A., Arszman, S. P., and Johns, E. S. (2007). Clinical characteristics of bipolar disorder in very young children. J. Affect. Disord. 97, 51-59.

Evans-Lacko, S., dosReis, S., Kastelic, E., Paula, C. S., and Steinwachs, D. M.
(2010). Evaluation of concordance with treatment guidelines for bipolar disorder among privately insured youth. Prim. Care Companion J. Clin. Psychiatry. 12, e1-e8.

Geller, B., Fox, L. W., and Clark, K. A. (1994). Rate and predictors of prepubertal bipolarity during follow-up of 6- to 12-year-old depressed children. J. Am. Acad. Child Adolesc. Psychiatry 33, 461-468.

Geller, B., Tillman, R., Bolhofner, K., and Zimerman,B.(2010). Pharmacological and non-drug treatment of child bipolar I disorder during prospective eight-year follow-up. Bipolar Disord 12, 164-171. 
Geller, B., Zimerman, B., Williams, M., Bolhofner, K., Craney, J. L., DelBello, M. P., and Soutullo, C. A. (2000). Diagnostic characteristics of 93 cases of a prepubertal and early adolescent bipolar disorder phenotype by gender, puberty and comorbid attention deficit hyperactivity disorder. $J$. Child Adolesc. Psychopharmacol. 10, 157-164.

Goodwin, F., and Jamison, K. (2007). Manic-Depressive Illness. New York: Oxford University Press.

Jerrell, J. M. (2008). Pharmacotherapy in the community-based treatment of children with bipolar I disorder. Hum. Psychopharmacol. 23, 53-59.

Jerrell, J. M., and Shugart, M. A. (2004). Community-based care for youths with early and very-early onset bipolar I disorder. Bipolar Disord. 6, 299-304.

Johns Hopkins. (2001). The Johns Hopkins ACG Case-Mix System ${ }^{\mathrm{TM}}$ : Documentation and Application Manual. Baltimore: The Johns Hopkins University.

Kashner,T.M.(1998). Agreement between administrative files and written medical records: a case of the Department of Veterans Affairs. Med. Care 36, 1324-1336.

Kowatch, R.A., and DelBello, M.P.(2005). Pharmacotherapy of children and adolescents with bipolar disorder. Psychiatr. Clin. North Am. 28, 385-397.

Leibenluft, E. (2006). Flying almost blind. Am. J. Psychiatry 163, 1129-1131.

Lurie, N., Popkin, M., Dysken, M., Moscovice, I., and Finch, M. (1992). Accuracy of diagnoses of schizophrenia in Medicaid claims. Hosp. Community Psychiatry 43, 69-71.

Moreno, C., Laje, G., Blanco, C., Jiang, H., Schmidt, A. B., and Olfson, M. (2007). National trends in the outpatient diagnosis and treatment of bipolar disorder in youth. Arch. Gen. Psychiatry 64, 1032-1039.

Mumford, L. M. (2008). Multum Lexicon Database. Cerner Multum. Available at: http://www.multum.com/Lexicon. htm (accessed July 25, 2008).

Post, R. M., and Kowatch, R. A. (2006) The health care crisis of childhoodonset bipolar illness: some recommendations for its amelioration. $J$. Clin. Psychiatry 67, 115-125.

Reid, R. J., MacWilliam, L., Verhulst, L., Roos, N., and Atkinson, M. (2001).
Performance of the ACG case-mix system in two Canadian provinces. Med. Care 39, 86-99.

Ryan, N. D., Puig-Antich, J., Ambrosini, P., Rabinovich, H., Robinson, D., Nelson, B., Iyengar, S., and Twomey, J. (1987). The clinical picture of major depression in children and adolescents. Arch. Gen. Psychiatry 44, 854-861.

Starfield, B. (2006). Threads and yarns: weaving the tapestry of comorbidity. Ann. Fam. Med. 4, 101-103.

Unutzer, J., Simon, G., Pabiniak, C., Bond, K., and Katon, W. (2000). The use of administrative data to assess quality of care for bipolar disorder in a large staff model HMO. Gen. Hosp. Psychiatry 22, $1-10$.

US Department of Health and Human Services. (2004). Area Resource File (ARF).Rockville,MD:HealthResources and Services Administration.

Weiner, J. P., Starfield, B. H., Steinwachs, D. M., and Mumford, L. M. (1991) Development and application of a population-oriented measure of ambulatory care case-mix. Med. Care 29, 452-472.

Wozniak, J. (2005). Recognizing and managing bipolar disorder in children. J. Clin. Psychiatry 66(Suppl. 1), 18-23.

Conflict of Interest Statement: The authors declare that the research was conducted in the absence of any commercial or financial relationships that could be construed as a potential conflict of interest.

Received: 22 July 2010; paper pending published: 28 September 2010; accepted: 07 October 2010; published online: 02 November 2010.

Citation: Evans-Lacko S, Riley AW and dosReis S (2010) Complexity and continuity of treatments among privately insured youth diagnosed with bipolar disorder. Front. Psychiatry 1:144. doi: 10.3389/ fpsyt.2010.00144

This article was submitted to Frontiers in Child and Neurodevelopmental Psychiatry, a specialty of Frontiers in Psychiatry.

Copyright (C) 2010 Evans-Lacko, Riley and dosReis. This is an open-access article subject to an exclusive license agreement between the authors and the Frontiers Research Foundation, which permits unrestricted use, distribution, and reproduction in any medium, provided the original authors and source are credited. 